\title{
QUANTUM TELEPORTATION AND QUANTUM EPISTEMIC SEMANTICS
}

\author{
Enrico Beltrametti* — Maria Luisa Dalla Chiara** - \\ Roberto Giuntini*** — Giuseppe Sergioli***
}

Dedicated to David Foulis

(Communicated by Sylvia Pulmannová)

\begin{abstract}
Quantum information gives rise to some puzzling epistemic problems that can be interestingly investigated from a logical point of view. A characteristic example is represented by teleportation phenomena, where knowledge and actions of observers (epistemic agents) play a relevant role. By abstracting from teleportation, we propose a simplified semantics for a language that consists of two parts:

1) the quantum computational sub-language, whose sentences $\alpha$ represent pieces of quantum information (which are supposed to be stored by some quantum systems);

2) the classical epistemic sub-language, whose atomic sentences have the following forms:

agent a has a probabilistic information about the sentence $\alpha$; agent a knows the sentence $\alpha$.
\end{abstract}

Interestingly enough, some conceptual difficulties of standard epistemic logics can be avoided in this framework.

$$
\begin{gathered}
\text { (C) } 2012 \\
\text { Mathematical Institute } \\
\text { Slovak Academy of Sciences }
\end{gathered}
$$

Quantum information gives rise to some intriguing epistemic situations that can be interestingly investigated from a logical point of view. As an example we can refer to the puzzling teleportation-case. By abstracting from the concrete quantum phenomena, we will propose a simplified semantics for a language that consists of two parts:

2010 Mathematics Subject Classification: Primary 81P10.

Keywords: quantum teleportation, quantum logic, epistemic logic.

The fourth author was supported by Regione Autonoma della Sardegna, POR Sardegna FSE-M.S. 2007-2013 L.R. 7/2007. 


\section{E. BELTRAMETTI - M. L. DALLA CHIARA - R. GIUNTINI - G. SERGIOLI}

1) the quantum computational sub-language, whose sentences $\alpha$ represent pieces of quantum information (which are supposed to be stored by some quantum systems);

2) the classical epistemic sub-language, whose atomic sentences have the following forms:

- agent a has a probabilistic information about the sentence $\alpha$;

- agent a knows the sentence $\alpha$.

We will see how some conceptual difficulties of standard epistemic logics can be avoided in this framework. We can mention for instance the unrealistic situations of epistemic logical omniscience, according to which knowing a given sentence should imply knowing all its logical consequences.

\section{Basis-changes and truth-perspectives}

Quantum computation and quantum computational logics are usually formulated by referring to the canonical orthonormal basis $B^{(1)}$ of the two dimensional Hilbert space $\mathbb{C}^{2}$. We have: $B^{(1)}=\{|0\rangle,|1\rangle\}$, where $|0\rangle=(1,0)$ and $|0\rangle=(0,1)$ represent the two classical bits in this context. By definition, a qubit is a unit vector of $\mathbb{C}^{2}$ whose canonical form is $|\psi\rangle=a|0\rangle+b|1\rangle$. Let $\mathcal{H}^{(n)}=\bigotimes^{n} \mathbb{C}^{2}$ be the $n$-fold tensor product of $\mathbb{C}^{2}$. The canonical basis of $\mathcal{H}^{(n)}$ is the set $B^{(n)}=\left\{\left|x_{1}\right\rangle \otimes \cdots \otimes\left|x_{n}\right\rangle:\left|x_{1}\right\rangle, \ldots,\left|x_{n}\right\rangle \in B^{(1)}\right\}$. As usual, we will briefly write $\left|x_{1}, \ldots, x_{n}\right\rangle$ instead of $\left|x_{1}\right\rangle \otimes \cdots \otimes\left|x_{n}\right\rangle$. By definition, a quregister is a unit vector of $\mathcal{H}^{(n)}$ whose canonical form is $|\psi\rangle=\sum_{i} a_{i}\left|x_{i_{1}}, \ldots, x_{i_{n}}\right\rangle$. Quregisters correspond to pure states: maximal pieces of knowledge about the quantum systems that are supposed to store a given amount of quantum information. In quantum computation one also needs mixtures of quregisters: density operators $\rho$ of $\mathcal{H}^{(n)}$, which will be briefly termed qumixes. We will denote by $\mathfrak{D}\left(\mathcal{H}^{(n)}\right)$ the set of all qumixes of $\mathcal{H}^{(n)}$, while $\mathfrak{D}=\bigcup_{n}\left\{\mathfrak{D}\left(\mathcal{H}^{(n)}\right)\right\}$ will represent the set of all possible qumixes.

One can wonder whether founding the semantics of quantum computational logics on the canonical bases of the spaces $\mathcal{H}^{(n)}$ might represent a logical limitation, since the choice of an orthonormal basis for a given Hilbert space is obviously conventional. What happens if the whole semantic construction is developed by taking as a starting point a different basis for $\mathbb{C}^{2}$ ? Any basis-change can be regarded as determined by a unitary operator $U$ of $\mathbb{C}^{2}$. As an example, let $U$ be the Hadamard operator $\sqrt{\mathrm{I}}$. Then, $U$ determines the following new orthonormal basis:

$$
B_{U}^{(1)}=\{\sqrt{\mathrm{I}}|0\rangle, \sqrt{\mathrm{I}}|1\rangle\}=\left\{\left(\frac{1}{\sqrt{ } 2}, \frac{1}{\sqrt{ } 2}\right),\left(\frac{1}{\sqrt{ } 2},-\frac{1}{\sqrt{ } 2}\right)\right\} .
$$




\section{QUANTUM TELEPORTATION AND QUANTUM EPISTEMIC SEMANTICS}

From an intuitive point of view, any basis-change can be imagined as a change of our truth-perspective. While in the canonical case, the truth-values truth and falsity are identified with the two classical bits $|1\rangle$ and $|0\rangle$, assuming a different basis corresponds to a different idea of truth and falsity. On this ground, one can guess that different epistemic agents (who may have different semantic ideas) are associated to different bases for the same space $\mathbb{C}^{2}$.

Any unitary operator $U$ of $\mathcal{H}^{(1)}$ can be canonically extended to a unitary operator $U^{(n)}$ of $\mathcal{H}^{(n)}$ (for any $n \geq 1$ ), which is defined as follows:

$$
U^{(n)}\left|x_{1}, \ldots, x_{n}\right\rangle=U\left|x_{1}\right\rangle \otimes \cdots \otimes U\left|x_{n}\right\rangle .
$$

Hence, any choice of a unitary operator $U$ of $\mathcal{H}^{(1)}$ determines an orthonormal basis $B_{U}^{(n)}$ for $\mathcal{H}^{(n)}$ such that:

$$
B_{U}^{(n)}=\left\{U^{(n)}\left|x_{1}, \ldots, x_{n}\right\rangle:\left|x_{1}, \ldots, x_{n}\right\rangle \in B^{(n)}\right\} .
$$

Instead of $U^{(n)}\left|x_{1}, \ldots, x_{n}\right\rangle$ we will also write $\left|x_{1}, \ldots, x_{n}\right\rangle_{U}$. Of course, we will have:

$$
U^{(n)^{-1}}\left|x_{1}, \ldots, x_{n}\right\rangle_{U}=\left|x_{1}, \ldots, x_{n}\right\rangle .
$$

The elements of $B_{U}^{(1)}$ are called the $U$-bits of $\mathcal{H}^{(1)}$, while the elements of $B_{U}^{(n)}$ are called the $U$-registers of $\mathcal{H}^{(n)}$.

Let $B_{U}$ represent the infinite sequence $B_{U}^{(1)}, B_{U}^{(2)}, \ldots$ that is determined by the unitary operator $U$. We will call $B_{U}$ a general basis, while $B$ will represent the canonical general basis. Of course, $B_{U}=B$ iff $U$ is the identity operator $I$.

For any choice of a general basis $B_{U}$, we define now the notions of truth, falsity and probability with respect to $B_{U}$.

Definition 1.1 (True and false registers).

- $\left|x_{1}, \ldots, x_{n}\right\rangle_{U}$ is a true register of $B_{U}^{(n)}$ iff $\left|x_{n}\right\rangle_{U}=|1\rangle_{U}$.

- $\left|x_{1}, \ldots, x_{n}\right\rangle_{U}$ is a false register of $B_{U}^{(n)}$ iff $\left|x_{n}\right\rangle_{U}=|0\rangle_{U}$.

In other words, the truth-value of an $U$-register (which corresponds to a sequence of $U$-bits) is determined by its last element 1

Let $\mathfrak{R}_{T}^{U}\left(\mathfrak{R}_{F}^{U}\right)$ represent the set of the true $U$-registers (the false $U$-registers) of $B_{U}^{(n)}$.

\footnotetext{
${ }^{1}$ As we will see, the application of a classical reversible gate to a register $\left|x_{1}, \ldots, x_{n}\right\rangle$ transforms the bit $\left|x_{n}\right\rangle$ into the target-bit $\left|x_{n}^{\prime}\right\rangle$, which behaves as the final truth-value. This justifies our choice in Definition 1.1
} 


\section{E. BELTRAMETTI - M. L. DALLA CHIARA - R. GIUNTINI - G. SERGIOLI}

Definition 1.2 (Truth and falsity).

- The $U$-truth of $\mathcal{H}^{(n)}$ is the projection operator $P_{1}^{U^{(n)}}$ that projects over the closed subspace spanned by $\mathfrak{R}_{T}^{U}$.

- The $U$-falsity of $\mathcal{H}^{(n)}$ is the projection operator $P_{0}^{U^{(n)}}$ that projects over the closed subspace spanned by $\mathfrak{R}_{F}^{U}$.

In this way, truth and falsity are dealt with as mathematical representatives of possible physical properties. Accordingly, by applying the Born-rule, one can naturally define the probability value of any qumix with respect to the general basis $B_{U}$.

Definition 1.3 (U-Probability).

For any $\rho \in \mathfrak{D}\left(\mathcal{H}^{(n)}\right)$,

$$
\mathrm{p}_{U}(\rho):=\operatorname{Tr}\left(P_{1}^{U^{(n)}} \rho\right),
$$

where $\operatorname{Tr}$ is the trace-functional.

In other words, $\mathrm{p}_{U}(\rho)$ represents the probability that the information $\rho$ satisfies the truth-property (with respect to the basis $B_{U}$ ). In the case of the canonical general basis we will write $\mathrm{p}(\rho)$ instead of $\mathrm{p}_{\mathrm{I}}(\rho)$.

As is well known, quantum information is processed by quantum logical gates (briefly, gates): unitary operators that transform quregisters into quregisters in a reversible way. We will first introduce some gates that play a special role both from the computational and from the logical point of view.

DeFinition 1.4 (The negation).

For any $n \geq 1$, the negation on $\mathcal{H}^{(n)}$ is the linear operator $\operatorname{NOT}^{(n)}$ such that, for every element $\left|x_{1}, \ldots, x_{n}\right\rangle$ of the basis $B^{(n)}$,

$$
\mathrm{NOT}^{(n)}\left(\left|x_{1}, \ldots, x_{n}\right\rangle\right)=\left|x_{1}, \ldots, x_{n-1}\right\rangle \otimes\left|1-x_{n}\right\rangle .
$$

In particular, we obtain:

$$
\mathrm{NOT}^{(1)}|0\rangle=|1\rangle ; \quad \mathrm{NOT}^{(1)}|1\rangle=|0\rangle,
$$

(according to the classical truth-table of negation).

Definition 1.5 (The Toffoli gate).

For any $n, m, p \geq 1$, the Toffoli gate is the linear operator $\mathrm{T}^{(n, m, p)}$ defined on $\mathcal{H}^{(n+m+p)}$ such that, for every element $\left|x_{1}, \ldots, x_{n}\right\rangle \otimes\left|y_{1}, \ldots, y_{m}\right\rangle \otimes\left|z_{1}, \ldots, z_{p}\right\rangle$ of the basis $B^{(n+m+p)}$,

$$
\begin{aligned}
\mathrm{T}^{(n, m, p)}\left(\left|x_{1}, \ldots, x_{n}\right\rangle\right. & \left.\otimes\left|y_{1}, \ldots, y_{m}\right\rangle \otimes\left|z_{1}, \ldots, z_{p}\right\rangle\right) \\
=\left|x_{1}, \ldots, x_{n}\right\rangle & \otimes\left|y_{1}, \ldots, y_{m}\right\rangle \otimes\left|z_{1}, \ldots, z_{p-1}, x_{n} y_{m} \widehat{+} z_{p}\right\rangle,
\end{aligned}
$$

where $\widehat{+}$ represents the addition modulo 2 . 


\section{QUANTUM TELEPORTATION AND QUANTUM EPISTEMIC SEMANTICS}

DEFINITION 1.6 (The XOR gate).

For any $n, m \geq 1$, the XOR gate is the linear operator XOR ${ }^{(n, m)}$ defined on $\mathcal{H}^{(n+m)}$ such that, for every element $\left|x_{1}, \ldots, x_{n}\right\rangle \otimes\left|y_{1}, \ldots, y_{m}\right\rangle$ of the basis $B^{(n+m)}$,

$$
\begin{aligned}
\operatorname{XOR}^{(n, m)} & \left(\left|x_{1}, \ldots, x_{n}\right\rangle \otimes\left|y_{1}, \ldots, y_{m}\right\rangle\right) \\
& =\left|x_{1}, \ldots, x_{n-1}\right\rangle \otimes\left|y_{1}, \ldots, y_{m-1}\right\rangle \otimes\left|x_{n}, x_{n} \widehat{+} y_{m}\right\rangle .
\end{aligned}
$$

In particular, we obtain:

$$
\mathrm{XOR}^{(1,1)}(|x, y\rangle)=|x, x \widehat{+} y\rangle .
$$

Hence, $\operatorname{XOR}^{(1,1)}$ behaves like a classical reversible exclusive disjunction (aut).

Definition 1.7 (The Hadamard-gate).

For any $n \geq 1$, the Hadamard-gate on $\mathcal{H}^{(n)}$ is the linear operator $\sqrt{\mathrm{I}}^{(n)}$ such that for every element $\left|x_{1}, \ldots, x_{n}\right\rangle$ of the basis $B^{(n)}$ :

$$
\sqrt{\mathrm{I}}^{(n)}\left(\left|x_{1}, \ldots, x_{n}\right\rangle\right)=\left|x_{1}, \ldots, x_{n-1}\right\rangle \otimes \frac{1}{\sqrt{2}}\left((-1)^{x_{n}}\left|x_{n}\right\rangle+\left|1-x_{n}\right\rangle\right) .
$$

In particular we obtain:

$$
\sqrt{\mathrm{I}}^{(1)}(|0\rangle)=\frac{1}{\sqrt{2}}(|0\rangle+|1\rangle) ; \quad \sqrt{\mathrm{I}}^{(1)}(|1\rangle)=\frac{1}{\sqrt{2}}(|0\rangle-|1\rangle) .
$$

Hence, $\sqrt{\mathrm{I}}^{(1)}$ transforms bits into genuine qubits.

Notice that the system consisting of the gates Negation, Toffoli, XOR and Hadamard is, to a certain extent, redundant. In fact, as proved by Shi and Aharanov ([10], [1]), Toffoli and Hadamard give rise to an approximately universal system of gates, in the sense that any gate $G$ (defined on $\mathcal{H}^{(n)}$ ) can be approximately simulated by a convenient combination of Toffoli and Hadamard up to an arbitrary accuracy. In this system Toffoli plays the role of a classical universal gate, which permits us to define exactly all classical reversible gates. For instance, the reversible conjunction AND and the reversible negative conjunction NAND can be defined as follows, for any $|\psi\rangle \in \mathcal{H}^{(n)}$ and any $|\varphi\rangle \in \mathcal{H}^{(m)}$ :

$$
\begin{aligned}
\operatorname{AND}(|\psi\rangle,|\varphi\rangle) & =\mathrm{T}^{(n, m, 1)}(|\psi\rangle \otimes|\varphi\rangle \otimes|0\rangle) ; \\
\operatorname{NAND}(|\psi\rangle,|\varphi\rangle) & =\mathrm{T}^{(n, m, 1)}(|\psi\rangle \otimes|\varphi\rangle \otimes|1\rangle) .
\end{aligned}
$$

The gate Hadamard, instead, represents a genuine quantum gate, that creates uncertain outputs (quregisters), starting from certain inputs (classical registers). Using independent definitions of NOT and of XOR is, however, more useful for computational aims, since such definitions permit us to avoid a non-economical increasing of the dimension of the Hilbert spaces in play.

All gates can be naturally transposed from the canonical (general) basis $B$ to the (general) basis $B_{U}$ that is determined by the unitary operator $U$. Let $G^{(n)}$ 


\section{E. BELTRAMETTI - M. L. DALLA CHIARA - R. GIUNTINI - G. SERGIOLI}

be any gate defined with respect to the canonical basis $B^{(n)}$. The twin-gate $G_{U}^{(n)}$, defined with respect to the basis $B_{U}^{(n)}$, is determined as follows:

$$
\forall\left|x_{1}, \ldots, x_{n}\right\rangle_{U} \in B_{U}^{(n)}: \quad G_{U}^{(n)}\left(\left|x_{1}, \ldots, x_{n}\right\rangle_{U}\right):=U^{(n)}\left(G^{(n)}\left(\left|x_{1}, \ldots, x_{n}\right\rangle\right)\right) .
$$

All $U$-gates can be canonically extended to the set $\mathfrak{D}$ of all qumixes. Let $G_{U}^{(n)}$ be any gate defined on $\mathcal{H}^{(n)}$. The corresponding qumix gate (also called unitary quantum operation ${ }^{\mathfrak{D}} G_{U}^{(n)}$ is defined as follows for any $\rho \in \mathfrak{D}\left(\mathcal{H}^{(n)}\right)$ :

$$
{ }^{\mathfrak{D}} G_{U}^{(n)}(\rho)=G_{U}^{(n)} \rho G_{U}^{(n)^{*}},
$$

where $G_{U}^{(n)^{*}}$ is the adjoint of $G_{U}^{(n)}$.

On this basis, one can uniformly define on the set $\mathfrak{D}$ a system of operations that correspond to the $U$-gates considered above.

Definition 1.8 (The operations Negation, Hadamard, Toffoli and XOR).

1. For any $\rho \in \mathfrak{D}\left(\mathcal{H}^{(n)}\right), \mathbb{N}_{U}(\rho)={ }^{\mathfrak{D}} \mathrm{NOT}_{U}^{(n)}(\rho)$.

2. For any $\rho \in \mathfrak{D}\left(\mathcal{H}^{(n)}\right), \sqrt{\mathbb{I}}_{U}(\rho)=\mathfrak{D} \sqrt{\mathrm{I}}_{U}^{(n)}(\rho)$.

3. For any $\rho \in \mathfrak{D}\left(\mathcal{H}^{(n)}\right)$, for any $\sigma \in \mathfrak{D}\left(\mathcal{H}^{(m)}\right)$ and for any $\tau \in \mathfrak{D}\left(\mathcal{H}^{(p)}\right)$, $\mathbb{T}_{U}(\rho, \sigma, \tau)={ }^{\mathfrak{D}} \mathrm{T}_{U}^{(n, m, p)}(\rho \otimes \sigma \otimes \tau)$.

4. For any $\rho \in \mathfrak{D}\left(\mathcal{H}^{(n)}\right)$ and for any $\sigma \in \mathfrak{D}\left(\mathcal{H}^{(m)}\right)$,

$$
\mathbb{X}_{U}(\rho, \sigma)={ }^{\mathfrak{D}} \mathrm{XOR}_{U}^{(n, m)}(\rho \otimes \sigma) \text {. }
$$

For any choice of a general basis $B_{U}$, the set $\mathfrak{D}$ can be pre-ordered by a relation that is defined in terms of the probability-function $\mathrm{p}_{U}$. We will see how this preorder will play an important role in the quantum computational semantics.

Definition 1.9 (U-Preorder).

$\rho \preceq_{U} \sigma \Longleftrightarrow \mathrm{p}_{U}(\rho) \leq \mathrm{p}_{U}(\sigma)$ and $\mathrm{p}_{U}\left(\sqrt{ }_{U}(\rho)\right) \leq \mathrm{p}_{U}\left(\sqrt{ } \mathbb{I}_{U}(\sigma)\right)$.

In the case of the canonical general basis, we will write $\rho \preceq \sigma$ instead of $\rho \preceq_{\text {I }} \sigma$.

We introduce now the notion of $U$-quantum computational structure, based on the set $\mathfrak{D}$ of all qumixes.

Definition 1.10 ( $U$-quantum computational structure).

Let $B_{U}$ be any general basis. The following structure

$$
\mathfrak{S}_{U}=\left(\mathfrak{D}, \preceq_{U}, \mathbb{N}_{U}, \sqrt{ } \mathbb{I}_{U}, \mathbb{T}_{U}, \mathbb{X}_{U}, P_{0}^{U}(1), P_{1}^{U(1)}\right)
$$

is called the $U$-quantum computational structure

By canonical quantum computational structure we mean the structure $\mathfrak{S}_{U}$ where $U$ is the identity operator $I$. 
TheOREM 1.1. Any U-computational structure is isomorphic to the canonical computational structure.

Proof. For any $n \geq 1$, consider the unitary quantum operation

$$
{ }^{\mathfrak{D}} U^{(n)}: \mathfrak{D}\left(\mathcal{H}^{(n)}\right) \rightarrow \mathfrak{D}\left(\mathcal{H}^{(n)}\right),
$$

such that for any $\rho \in \mathfrak{D}\left(\mathcal{H}^{(n)}\right),{ }^{\mathfrak{D}} U^{(n)}(\rho)=U^{(n)} \rho U^{(n)^{*}}$. Consider then the following operation that is uniformly defined on the set $\mathfrak{D}$ :

$$
\forall \rho \in \mathfrak{D}\left(\mathcal{H}^{(n)}\right): \quad \mathbb{U}(\rho)={ }^{\mathfrak{D}} U^{(n)}(\rho) .
$$

We have:

(1) $\mathbb{U}$ is a bijection.

(2) $\forall \rho \in \mathfrak{D}: \quad \mathrm{p}(\rho)=\mathrm{p}_{U}(\mathbb{U}(\rho))$

(3) $\mathbb{U}(\mathbb{N}(\rho))=\mathbb{N}_{U}(\mathbb{U}(\rho))$.

(4) $\mathbb{U}(\sqrt{\mathbb{I}}(\rho))=\sqrt{\mathbb{I}}_{U}(\mathbb{U}(\rho))$.

(5) $\mathbb{U}(\mathbb{T}(\rho, \sigma, \tau))=\mathbb{T}_{U}(\mathbb{U}(\rho), \mathbb{U}(\sigma), \mathbb{U}(\tau))$.

(6) $\mathbb{U}(\mathbb{X}(\rho, \sigma))=\mathbb{X}_{U}(\mathbb{U}(\rho), \mathbb{U}(\sigma))$.

(7) $\rho \preceq \sigma \Longleftrightarrow \mathbb{U}(\rho) \preceq U \mathbb{U}(\sigma)$.

\section{Holistic semantics and absolute quantum computational logic}

Quantum computational logics (QCL's) represent a natural logical abstraction from quantum computational circuits 2 In these logics sentences $\alpha$ are supposed to denote pieces of quantum information (generally qumixes), living in a Hilbert space whose dimension depends on the linguistic complexity of $\alpha$. At the same time, the logical connectives correspond to qumix gates that are processing the qumixes expressed by the sentences in question.

Let us first introduce a formal (sentential) language $\mathcal{L}^{Q C}$ for $\mathbf{Q} \mathbf{C L}^{\prime} \mathbf{s}$. This language contains atomic sentences (say, "the spin-value in the $x$-direction is up"), including two privileged sentences $\mathbf{t}$ and $\mathbf{f}$ that represent the truth-values Truth and Falsity, respectively. We will use $\mathbf{q}, \mathbf{r}, \ldots$ as metavariables for atomic sentences, and $\alpha, \beta, \ldots$ as metavariables for sentences. The connectives of $\mathcal{L}^{Q C}$ are: the negation $\neg$ (which corresponds to the qumix gate Negation), the squareroot of the identity $\sqrt{\mathrm{id}}$ (which corresponds to Hadamard), a ternary conjunction $\bigwedge$ (which corresponds to the reversible conjunction defined in terms of Toffoli), the exclusive disjunction $\biguplus$ (which corresponds to XOR), the composition-connective $\sharp$

${ }^{2}$ See [6], 4]. 


\section{E. BELTRAMETTI - M. L. DALLA CHIARA - R. GIUNTINI - G. SERGIOLI}

(which describes compositions of quantum systems). For any sentences $\alpha$ and $\beta$, the expressions $\neg \alpha, \sqrt{\mathrm{id}} \alpha, \bigwedge(\alpha, \beta, \mathbf{f})$ (the ternary conjunction of $\alpha, \beta, \mathbf{f}$ ), $\alpha \biguplus \beta$ are sentences. For any sentences $\beta_{1}, \ldots, \beta_{n}$, the expression $\sharp\left(\beta_{1}, \ldots, \beta_{n}\right)$ (the composition of $\beta_{1}, \ldots \beta_{n}$ ) is a sentence. The connectives $\neg, \sqrt{\mathrm{id}}, \Lambda, \biguplus$ are called gate-connectives. We will use the following metalinguistic abbreviations:

$$
\alpha \wedge \beta:=\bigwedge(\alpha, \beta, \mathbf{f}) ; \quad \beta_{1} \sharp \ldots \sharp \beta_{n}:=\sharp\left(\beta_{1}, \ldots, \beta_{n}\right) .
$$

Before defining the basic notions of the quantum computational semantics, let us first introduce some useful syntactical notions.

\section{DEFINITION 2.1.}

- $\alpha$ is called a gate-sentence iff either $\alpha$ is atomic or the principal connective of $\alpha$ is a gate-connective.

- $\alpha$ is called a compositional sentence iff $\alpha=\beta_{1} \sharp \ldots \sharp \beta_{m}$, where $\beta_{1}, \ldots, \beta_{m}$ are gate-sentences.

Definition 2.2 (The atomic complexity of a sentence).

The atomic complexity $\operatorname{At}(\alpha)$ of a sentence $\alpha$ is the number of occurrences of atomic sentences in $\alpha$.

For instance, $\operatorname{At}(\bigwedge(\mathbf{q}, \mathbf{q}, \mathbf{f}))=3$. We will also indicate by $\alpha^{(n)}$ a sentence whose atomic complexity is $n$. The notion of atomic complexity plays an important semantic role. For, the meaning of any sentence whose atomic complexity is $n$ is supposed to live in the domain $\mathcal{H}^{(n)}$. For this reason, $\mathcal{H}^{(\operatorname{At}(\alpha) \text { ) }}$ (briefly indicated by $\mathcal{H}^{\alpha}$ ) will be also called the semantic space of $\alpha$.

Any sentence $\alpha$ can be naturally decomposed into its parts, giving rise to a special configuration called the syntactical tree of $\alpha$ (indicated by STree ${ }^{\alpha}$ ).

Roughly, STree $^{\alpha}$ can be represented as a finite sequence of levels:

$$
\begin{gathered}
\operatorname{Level}_{k}(\alpha) \\
\vdots \\
\operatorname{Level}_{1}(\alpha),
\end{gathered}
$$

where:

- each $\operatorname{Level}_{i}(\alpha)$ (with $1 \leq i \leq k$ ) is a compositional sentence $\beta_{1} \sharp \ldots \sharp \beta_{m}$ that contains the atomic sentences of $\alpha$;

- the bottom level $\left(\operatorname{Level}_{1}(\alpha)\right)$ is $\alpha$;

- the top level $\left(\operatorname{Level}_{k}(\alpha)\right)$ is the sentence $\mathbf{q}_{1} \sharp \ldots \sharp \mathbf{q}_{t}$, where $\mathbf{q}_{1}, \ldots \mathbf{q}_{t}$ are the atomic occurrences in $\alpha$; 
- for any $i$ (with $1 \leq i<k$ ), $\operatorname{Level}_{i+1}(\alpha)$ is the compositional sentence obtained by dropping the principal gate-connective in all molecular gatesentences occurring at $\operatorname{Level}_{i}(\alpha)$, and by repeating all the atomic sentences that possibly occur at $\operatorname{Level}_{i}(\alpha)$.

By Height of $\alpha$ (indicated by Height $(\alpha)$ ) we mean the number of levels of the syntactical tree of $\alpha$.

As an example, consider the following sentence: $\alpha=\mathbf{q} \wedge \neg \mathbf{q}=\bigwedge(\mathbf{q}, \neg \mathbf{q}, \mathbf{f})$. The syntactical tree of $\alpha$ is the following sequence of levels:

$$
\begin{aligned}
& \operatorname{Level}_{3}(\alpha)=\mathbf{q} \sharp \mathbf{q} \sharp \mathbf{f} ; \\
& \operatorname{Level}_{2}(\alpha)=\mathbf{q} \sharp \neg \mathbf{q} \sharp \mathbf{f} ; \\
& \operatorname{Level}_{1}(\alpha)=\bigwedge(\mathbf{q}, \neg \mathbf{q}, \mathbf{f}) .
\end{aligned}
$$

Clearly, Height $(\bigwedge(\mathbf{q}, \neg \mathbf{q}, \mathbf{f}))=3$.

More precisely, the syntactical tree of a sentence (whose atomic complexity is $t$ ) is defined as follows.

Definition 2.3 (The syntactical tree of $\alpha$ ). The syntactical tree of $\alpha$ is the following sequence of sentences:

$$
\text { STree }^{\alpha}=\left(\operatorname{Level}_{1}(\alpha), \ldots, \operatorname{Level}_{k}(\alpha)\right),
$$

where:

- $\operatorname{Level}_{1}(\alpha)=\alpha$;

- Level $_{i+1}$ is defined as follows for any $i$ such that $1 \leq i<k$. The following cases are possible:

(1) $\operatorname{Level}_{i}(\alpha)$ does not contain any gate-connective.

Hence, $\operatorname{Level}_{i}(\alpha)=\mathbf{q}_{1} \sharp \ldots \sharp \mathbf{q}_{t}$ and Height $(\alpha)=i$;

(2) $\operatorname{Level}_{i}(\alpha)$ is the compositional sentence $\beta_{1} \sharp \ldots \sharp \beta_{m}$, and for at least one $j$, the principal connective of $\beta_{j}$ is a gate-connective. Consider the following sequence of sentences:

$$
\beta_{1}^{\prime}, \ldots, \beta_{m}^{\prime}
$$

such that:

$$
\beta_{h}^{\prime}= \begin{cases}\beta_{h}, & \text { if } \beta_{h} \text { is atomic } \\ \beta_{h}^{*}, & \text { otherwise }\end{cases}
$$

where:

$$
\beta_{h}^{*}= \begin{cases}\delta, & \text { if } \beta_{h}=\neg \delta \text { or } \beta_{h}=\sqrt{\text { id }} \delta ; \\ \gamma \sharp \delta \sharp \mathbf{f}, & \text { if } \beta_{h}=\bigwedge(\gamma, \delta, \mathbf{f}) ; \\ \gamma \sharp \delta, & \text { if } \beta_{h}=\gamma \biguplus \delta .\end{cases}
$$




\section{E. BELTRAMETTI - M. L. DALLA CHIARA - R. GIUNTINI - G. SERGIOLI}

Then,

$$
\operatorname{Level}_{i+1}(\alpha)=\beta_{1}^{\prime} \sharp \ldots \sharp \beta_{m}^{\prime} .
$$

The syntactical tree of $\alpha$ (which represents a purely syntactical object) uniquely determines a sequence of qumix gates that are all defined on the semantic space of $\alpha$, for any choice of a general basis $B_{U}$. This gate-sequence is called the $U$-qumix tree of $\alpha$.

As an example, consider again the sentence $\alpha=\bigwedge(\mathbf{q}, \neg \mathbf{q}, \mathbf{f})$ and its syntactical tree. Apparently, $\operatorname{Level}_{2}(\alpha)$ is obtained from $\operatorname{Level}_{3}(\alpha)$ by repeating the first occurrence of $\mathbf{q}$, by negating the second occurrence of $\mathbf{q}$ and by repeating $\mathbf{f}$. Hence the qumix gate that corresponds to $\operatorname{Level}_{2}(\alpha)$ will naturally be ${ }^{\mathfrak{D}} \mathrm{I}^{(1)} \otimes$ ${ }^{\mathfrak{D}} \mathrm{NOT}_{U}^{(1)} \otimes{ }^{\mathfrak{D}} \mathrm{I}^{(1)}$. Finally, $\operatorname{Level}_{1}(\alpha)$ is obtained from $\operatorname{Level}_{2}(\alpha)$ by applying to the three sentences occurring at $\operatorname{Level}_{2}(\alpha)$ the ternary conjunction. Hence the qumix gate that corresponds to $\operatorname{Level}_{1}(\alpha)$ will naturally be ${ }^{\mathfrak{D}} \mathrm{T}^{(1,1,1)}$. On this basis, the $U$-qumix tree of the sentence $\alpha=\bigwedge(\mathbf{q}, \neg \mathbf{q}, \mathbf{f})$ can be identified with the following sequence consisting of two $U$-qumix gates:

$$
\left({ }^{\mathfrak{D}} \mathrm{I}^{(1)} \otimes{ }^{\mathfrak{D}} \mathrm{NOT}_{U}^{(1)} \otimes{ }^{\mathfrak{D}} \mathrm{I}^{(1)},{ }^{\mathfrak{D}} \mathrm{T}_{U}^{(1,1,1)}\right) .
$$

The general definition of $U$-qumix tree is the following:

Definition 2.4 (The $U$-qumix tree of $\alpha$ ).

Let $\alpha$ be a sentence such that $\operatorname{Height}(\alpha)=k$. The $U$-qumix tree of $\alpha$ is the sequence of qumix gates

$$
\text { QumTree }_{U}^{\alpha}=\left({ }^{\mathfrak{D}} G_{U_{(k-1)}}^{\alpha}, \ldots,{ }^{\mathfrak{D}} G_{U_{(1)}}^{\alpha}\right),
$$

that is defined as follows. Suppose that

$$
\operatorname{Level}_{i-1}(\alpha)=\beta_{1}^{\left(t_{1}\right)} \sharp \ldots \sharp \beta_{m}^{\left(t_{m}\right)},
$$

(where $1 \leq i \leq k)$. We put:

$$
{ }^{\mathfrak{D}} G_{U_{(i-1)}}^{\alpha}={ }^{\mathfrak{D}} X_{U_{(1)}^{\left(t_{1}\right)}} \otimes \cdots \otimes \otimes^{\mathfrak{D}} X_{U_{(m)}}^{\left(t_{m}\right)},
$$

where any ${ }^{\mathfrak{D}} X_{U_{(j)}}^{\left(t_{j}\right)}$ is a qumix gate defined on $\mathcal{H}^{\left(t_{j}\right)}$ such that:

$$
{ }^{\mathfrak{D}} X_{U}^{\left(t_{j}\right)}= \begin{cases}\mathfrak{D} \mathrm{I}^{\left(t_{j}\right)}, & \text { if } \beta_{j}^{\left(t_{j}\right)} \text { is atomic; } \\ \mathfrak{D}^{\mathfrak{D} O \mathrm{~T}_{U}^{\left(t_{j}\right)},}, & \text { if } \beta_{j}^{\left(t_{j}\right)}=\neg \delta ; \\ \mathfrak{D} \sqrt{\mathrm{I}_{U}^{\left(t_{j}\right)},}, & \text { if } \beta_{j}^{\left(t_{j}\right)}=\sqrt{\mathrm{id}} \delta ; \\ \mathfrak{D} \mathrm{T}_{U}^{(r, s, 1)}, & \text { if } \beta_{j}^{\left(t_{j}\right)}=\bigwedge\left(\gamma^{(r)}, \delta^{(s)}, \mathbf{f}\right) ; \\ \mathfrak{D} \mathrm{XOR}_{U}^{(r, s)}, & \text { if } \beta_{j}^{\left(t_{j}\right)}=\gamma^{(r)} \biguplus \delta^{(s)} .\end{cases}
$$


Consider now a sentence $\alpha$ and let $\left({ }^{\mathfrak{D}} G_{U_{(k-1)}}^{\alpha}, \ldots,{ }^{\mathfrak{D}} G_{U_{(1)}}^{\alpha}\right)$ be the $U$-qumix tree of $\alpha$. Any choice of a qumix $\rho$ in $\mathcal{H}^{\alpha}$ determines a sequence $\left(\rho_{k}, \ldots, \rho_{1}\right)$ of qumixes of $\mathfrak{D}^{\alpha}$, where:

$$
\begin{aligned}
\rho_{k} & =\rho \\
\rho_{k-1} & ={ }^{\mathfrak{D}} G_{U_{(k-1)}}^{\alpha}\left(\rho_{k}\right) \\
& \vdots \\
\rho_{1} & ={ }^{\mathfrak{D}} G_{U_{(1)}}^{\alpha}\left(\rho_{2}\right) .
\end{aligned}
$$

The qumix $\rho_{k}$ can be regarded as a possible input-information concerning the atomic parts of $\alpha$, while $\rho_{1}$ represents the output-information about $\alpha$, given the input-information $\rho_{k}$. Each $\rho_{i}$ corresponds to the information about $\operatorname{Level}_{i}(\alpha)$, given the input-information $\rho_{k}$.

How to determine an information about the parts of $\alpha$ under a given input? It is natural to apply the reduced state function that (according to the quantum theoretic formalism) determines for any state $\rho$ of a composite system $S=S_{1}+\cdots+S_{n}$ the state $\operatorname{Red}^{i_{1}, \ldots, i_{m}}(\rho)$ of any subsystem $S_{i_{1}}+\cdots+S_{i_{m}}$ (where $1 \leq i_{1} \leq n, \ldots, 1 \leq i_{m} \leq n$.) As is well known, a characteristic case that arises in entanglement-phenomena is the following: while $\rho$ (the state of the global system) is pure (a maximal information), the reduced state $\operatorname{Red}^{i_{1}, \ldots, i_{m}}(\rho)$ is generally a mixture (a non-maximal information). Hence our information about the whole cannot be reconstructed as a function of our pieces of information about the parts. Such situations play a relevant role in the quantum computational semantics.

Consider the syntactical tree of $\alpha$ and suppose that:

$$
\operatorname{Level}_{i}(\alpha)=\beta_{i_{1}} \sharp \ldots \sharp \beta_{i_{r}} .
$$

We know that the $U$-qumix tree of $\alpha$ and the choice of an input $\rho_{k}$ (in $\mathcal{H}^{\alpha}$ ) determine a sequence of qumixes:

$$
\begin{gathered}
\rho_{k} \leadsto \operatorname{Level}_{k}(\alpha)=\mathbf{q}_{1} \sharp \ldots \sharp \mathbf{q}_{t} \\
\vdots \\
\rho_{i} \leadsto \operatorname{Level}_{i}(\alpha)=\beta_{i_{1} \sharp \ldots \sharp \beta_{i_{r}}} \\
\vdots \\
\rho_{1} \leadsto \operatorname{Level}_{1}(\alpha)=\alpha
\end{gathered}
$$




\section{E. BELTRAMETTI - M. L. DALLA CHIARA - R. GIUNTINI - G. SERGIOLI}

We can consider $\operatorname{Red}^{j}\left(\rho_{i}\right)$, the reduced information of $\rho_{i}$ with respect to the $j$ th part. From a semantic point of view, this object can be regarded as a contextual information about $\beta_{i_{j}}$ (the subformula of $\alpha$ occurring at the $j$ th position at $\left.\operatorname{Level}_{i}(\alpha)\right)$ under the input $\rho_{k}$.

We will now give the basic definitions of our semantics. The main concept is the notion of quantum computational model: a map that assigns to any sentence $\alpha$ of the language $\mathcal{L}^{Q C}$ a meaning, a qumix living in the semantic space $\mathcal{H}^{\alpha}$. Of course (like in the standard semantic approaches), the map shall respect the logical form of $\alpha$. We will consider a holistic version of semantics, which has been naturally inspired by the characteristic holistic features of the quantum theoretic formalism. In this semantics any model assigns to any sentence a global meaning that determines the contextual meanings of all its parts (from the whole to the parts!). It may happen that one and the same sentence receives different meanings in different contexts.

We will distinguish two possible "levels of semantic holism", which correspond to different notions of holistic model. Accordingly, we will speak of superholistic semantics and of normal holistic semantics, respectively. The superholistic semantics is the most liberal one: sentences may receive different meanings even in the framework of one and the same context. In other words, different occurrences of one and the same subformula in a given sentence may have different contextual meanings. Such a liberal point of view might appear somewhat strange in the case of scientific languages. However, it is quite reasonable for natural and artistic languages. Consider a very "long" expression $\gamma$ (for instance, a novel or a poem): why should all occurrences of a part of $\gamma$ have a constant contextual meaning? The normal holistic semantics is more restrictive: although sentences may receive different meanings in different contexts, all occurrences of a subformula in a given sentence receive a constant contextual meaning 3

Definition 2.5 (Superholistic model).

A superholistic model of the language $\mathcal{L}^{Q C}$ with respect to the general basis $B_{U}$ is a map $\mathrm{Hol}_{U}$ that associates a meaning $\operatorname{Hol}_{U}\left(\operatorname{Level}_{i}(\alpha)\right)$ to each level of the syntactical tree of $\alpha$, for any sentence $\alpha$ of $\mathcal{L}^{Q C}$. The following conditions are required:

(1) $\operatorname{Hol}_{U}\left(\operatorname{Level}_{i}(\alpha)\right) \in \mathfrak{D}\left(\mathcal{H}^{\alpha}\right)$.

In other words, the meaning of $\operatorname{Level}_{i}(\alpha)$ under $\mathrm{Hol}_{U}$ belongs to the semantic space of $\alpha$.

\footnotetext{
${ }^{3}$ We extend here to any general basis the semantic notions, defined in 4 with respect to the canonical basis.
} 


\section{QUANTUM TELEPORTATION AND QUANTUM EPISTEMIC SEMANTICS}

(2) Let $\left({ }^{\mathfrak{D}} G_{U_{(\operatorname{Height}(\alpha)-1)}^{\alpha}}^{\alpha}, \ldots,{ }^{\mathfrak{D}} G_{U_{(1)}}^{\alpha}\right)$ be the $U$-qumix tree of $\alpha$ and let $1 \leq i<\operatorname{Height}(\alpha)$. Then,

$$
\operatorname{Hol}_{U}\left(\operatorname{Level}_{i}(\alpha)\right)={ }^{\mathfrak{D}} G_{U_{(i)}}^{\alpha}\left(\operatorname{Hol}_{U}\left(\operatorname{Level}_{i+1}(\alpha)\right)\right) .
$$

In other words the global meaning of each level (different from the top level) is obtained by applying the corresponding gate to the meaning of the level that occurs immediately above.

(3) Let $\operatorname{Level}_{i}(\alpha)=\beta_{1} \sharp \ldots \sharp \beta_{r}$. Then:

$\beta_{j}=\mathbf{f} \curvearrowright \operatorname{Red}^{j}\left(\operatorname{Hol}_{U}\left(\operatorname{Level}_{i}(\alpha)\right)\right)=P_{0}^{U(1)} ;$

$\beta_{j}=\mathbf{t} \curvearrowright \operatorname{Red}^{j}\left(\operatorname{Hol}_{U}\left(\operatorname{Level}_{i}(\alpha)\right)\right)=P_{1}^{U(1)}$, for any $j(1 \leq j \leq r)$.

In other words, the contextual meanings of $\mathbf{f}$ and of $\mathbf{t}$ are always the Falsity and the Truth, respectively.

On this basis, we put:

$$
\operatorname{Hol}_{U}(\alpha):=\operatorname{Hol}_{U}\left(\operatorname{Level}_{1}(\alpha)\right),
$$

for any sentence $\alpha$.

Unlike standard compositional semantics, any $\operatorname{Hol}_{U}(\alpha)$ represents a kind of autonomous semantic context that is not necessarily correlated with the meanings of other sentences. At the same time, given a sentence $\gamma, \mathrm{Hol}_{U}$ determines the contextual meaning, with respect to the context $\mathrm{Hol}_{U}(\gamma)$, of any occurrence of a subformula $\beta$ in $\gamma$.

Definition 2.6 (Contextual meaning of a subformula). Let $\beta$ be a subformula of $\gamma$.

1. Suppose that $\beta$ is a gate-sentence and let $\beta\left[\begin{array}{l}i \\ j\end{array}\right]$ be an occurrence of $\beta$ at the $j$ th-position of the $i$ th-level of the syntactical tree of $\gamma$. Then

$$
\operatorname{Hol}_{U}^{\gamma}\left(\beta\left[\begin{array}{l}
i \\
j
\end{array}\right]\right):=\operatorname{Red}^{j}\left(\operatorname{Hol}_{U}\left(\operatorname{Level}_{i}(\gamma)\right)\right) .
$$

2. Suppose that $\beta$ is a compositional sentence occurring as a subformula at the $i$ th-level of the syntactical tree of $\gamma$. In other words, $\beta$ has the form $\beta_{1} \sharp \ldots \sharp \beta_{m}$ and $\operatorname{Level}_{i}(\gamma)=\delta_{1} \sharp \ldots \sharp \beta_{1}\left[\begin{array}{l}i_{j_{1}} \\ ]\end{array}\right] \sharp \ldots \sharp \beta_{m}\left[\begin{array}{l}i \\ j_{m}\end{array}\right] \sharp \ldots \sharp \delta_{r}$. Then,

$$
\operatorname{Hol}_{U}^{\gamma}\left(\beta_{1}\left[\begin{array}{l}
i \\
j_{1}
\end{array}\right] \sharp \ldots \sharp \beta_{m}\left[\begin{array}{l}
i \\
j_{m}
\end{array}\right]\right)=\operatorname{Red}^{j_{1}, \ldots, j_{m}}\left(\operatorname{Hol}_{U}\left(\operatorname{Level}_{i}(\gamma)\right)\right) \text {. }
$$

Hence, in particular, we have for any sentence $\gamma$

$$
\operatorname{Hol}_{U}^{\gamma}(\gamma)=\operatorname{Hol}_{U}\left(\operatorname{Level}_{1}(\gamma)\right)=\operatorname{Hol}_{U}(\gamma) \text {. }
$$

Apparently, $\operatorname{Hol}_{U}^{\gamma}$ is a partial function that only assigns meanings to the occurrences of subformulas of $\gamma$. Given a formula $\gamma$, we will call the partial function $\mathrm{Hol}_{U}^{\gamma}$ a contextual holistic model of the language. 


\section{E. BELTRAMETTI - M. L. DALLA CHIARA - R. GIUNTINI - G. SERGIOLI}

Definition 2.7 (Normal holistic model).

A normal holistic model of the language $\mathcal{L}^{Q C}$ is a superholistic model Hol ${ }_{U}$ that assigns to any occurrence of a subformula $\beta$ of $\gamma$ in the syntactical tree of $\gamma$ the same contextual meaning, which will be uniformly indicated by $\operatorname{Hol}_{U}^{\gamma}(\beta)$.

In the following we will always refer to normal holistic models with respect to a general basis $B_{U}$ (briefly called $U$-holistic models).

Suppose that $\beta$ is a subformula of two different formulas $\gamma$ and $\delta$. Generally, we have:

$$
\operatorname{Hol}_{U}^{\gamma}(\beta) \neq \operatorname{Hol}_{U}^{\delta}(\beta) .
$$

In other words, sentences may receive different contextual meanings in different contexts also in the case of the normal holistic semantics.

We can now define the notions of truth and logical consequence.

Definition 2.8 (Truth).

A sentence $\alpha$ is true in the model $\mathrm{Hol}_{U}$ (abbreviated as $\models_{\mathrm{Hol}_{U}} \alpha$ ) iff

$$
\mathrm{p}_{U}\left(\operatorname{Hol}_{U}(\alpha)\right)=1 .
$$

When $U=\mathrm{I}$ we will speak of canonical truth.

Definition 2.9 (Logical consequence).

A sentence $\beta$ is a logical consequence of a sentence $\alpha$ with respect to the general basis $B_{U}\left(\alpha \models{ }_{B_{U}} \beta\right)$ iff for any sentence $\gamma$ such that $\alpha$ and $\beta$ are subformulas of $\gamma$ and for any model $\operatorname{Hol}_{U}$, $\operatorname{Hol}_{U}^{\gamma}(\alpha) \preceq_{U} \operatorname{Hol}_{U}^{\gamma}(\beta)$ (where $\preceq_{U}$ is the preorder relation defined in Section 1).

On this basis, we can semantically characterize a particular form of quantum computational logic that will be termed absolute quantum computational logic (AbsQCL).

Definition 2.10 (Absolute quantum computational logic).

A sentence $\beta$ is a logical consequence of a sentence $\alpha$ in the logic AbsQCL $(\alpha \models \mathbf{A b s Q C L} \beta)$ iff for any general basis $B_{U}, \alpha \models_{B_{U}} \beta$.

While the concepts of probability and of truth are obviously dependent on the choice of the general basis $B_{U}$, one can prove that the notion of logical consequence is invariant with respect to any basis-change.

TheOREM 2.1. $\alpha \models_{\text {AbsQCL }} \beta$ iff $\alpha \models_{B_{\mathrm{I}}} \beta$.

Proof. The left-right arrow is trivial. In order to prove the right-left arrow, suppose that there exist two sentences $\alpha$ and $\beta$ such that: $\alpha \models_{B_{I}} \beta$ and $\alpha \not \models$ AbsQCL $\beta$. Then, there exist a formula $\gamma$ including $\alpha$ and $\beta$ as subformulas, a general basis $B_{U}$ and a model $\mathrm{Hol}_{U}$ such that:

$$
\operatorname{Hol}_{U}^{\gamma}(\alpha) \npreceq U \operatorname{Hol}_{U}^{\gamma}(\beta) \text {. }
$$


Define a canonical model Hol $\mathrm{I}$ as follows: for any sentence $\delta, \operatorname{Hol}_{\mathrm{I}}\left(\operatorname{Level}_{i}(\delta)\right)=$ $\mathbb{U}^{-1}\left(\operatorname{Hol}_{U}\left(\operatorname{Level}_{i}(\delta)\right)\right.$ ) (where $\mathbb{U}$ is the map defined in the proof of Theorem 1.1). As a consequence, we have: $\operatorname{Hol}_{\mathrm{I}}(\gamma)=\mathbb{U}^{-1}\left(\operatorname{Hol}_{U}(\gamma)\right) ; \operatorname{Hol}_{\mathrm{I}}^{\gamma}(\alpha)=\mathbb{U}^{-1}\left(\operatorname{Hol}_{U}^{\gamma}(\alpha)\right)$; $\operatorname{Hol}_{\mathrm{I}}^{\gamma}(\beta)=\mathbb{U}^{-1}\left(\operatorname{Hol}_{U}^{\gamma}(\beta)\right)$. Hence, by Theorem 1.1 $\operatorname{Hol}_{\mathrm{I}}^{\gamma}(\alpha) \npreceq \operatorname{Hol}_{\mathrm{I}}^{\gamma}(\beta)$. Against the hypothesis.

\section{Epistemic situations in quantum teleportation}

We will now briefly illustrate the puzzling epistemic situations that arise in quantum teleportation phenomena. Two observers, Alice and Bob (also called epistemic agents) have a partial access to a composite quantum system $S=$ $S_{1}+S_{2}+S_{3}$, consisting of three particles (say, three photons). At any time $t_{i}$ of a given time-sequence $\left(t_{o}, \ldots, t_{n}\right)$, both of them have a global or a partial information about the state of $S$. Furthermore, both of them can modify the state of $S$ (or of a subsystem of $S$ ) either by applying some quantum logical gates or by performing a measurement (which induces a collapse of the wave function).

Alice and $B o b$ are supposed to have interacted in the past with the composite system $S_{2}+S_{3}$, whose parts are separated at time $t_{0}: S_{2}$ is accessible to Alice, while $S_{3}$ is accessible to $B o b$. As a consequence, at time $t_{0}$, Alice and $B o b$ have the same information about the state of the subsystem $S_{2}+S_{3}$, which is supposed to be entangled:

$$
\left|\psi^{S_{2}+S_{3}}\right\rangle\left(t_{0}\right)=\frac{1}{\sqrt{2}}(|0,0\rangle+|1,1\rangle) .
$$

In other words, at time $t_{0}$ both agents know that two cases are possible with equal probability $\left(\frac{1}{2}\right)$ :

1. The state of both parts $\left(S_{2}, S_{3}\right)$ is the bit $|0\rangle$.

2. The state of both parts $\left(S_{2}, S_{3}\right)$ is the bit $|1\rangle$.

At time $t_{0}$, Alice has also access to the first particle $S_{1}$, whose state is the qubit

$$
\left|\psi^{S_{1}}\right\rangle\left(t_{0}\right)=a|0\rangle+b|1\rangle \text {. }
$$

The epistemic situation of either agent at any time $t$ (of the considered timesequence) can be represented as a pair consisting of

a) the physical system $S_{\text {Agent }_{t}}$ that is physically accessible to our agent;

b) the state $\rho_{\text {Agent }_{t}}$ that represents the agent's information about a given system (which may include $S_{\text {Agent }_{t}}$ as a subsystem). 


\section{E. BELTRAMETTI - M. L. DALLA CHIARA - R. GIUNTINI - G. SERGIOLI}

We will write:

$$
\operatorname{Inf}\left(\text { Agent }_{t}\right)=\left(S_{\text {Agent }_{t}}, \rho_{\text {Agent }_{t}}\right) .
$$

Notice that the "theoretic" information $\rho_{\text {Agent }_{t}}$ concerns a system that is generally larger than the system accessible to our agent at time $t$.

We can now represent the epistemic situations of Alice and Bob at time $t_{0}$ as follows.

\section{Alice at time $t_{0}$}

$$
\operatorname{Inf}\left(\text { Alice }_{t_{0}}\right)=\left(\left(S_{1}+S_{2}\right)_{t_{0}},\left|\psi^{S}\right\rangle_{t_{0}}\right)
$$

where

$$
\begin{aligned}
\left|\psi^{S}\right\rangle_{t_{0}} & =(a|0\rangle+b|1\rangle) \otimes \frac{1}{\sqrt{2}}(|0,0\rangle+|1,1\rangle) \\
& =\frac{1}{\sqrt{2}}\left(a|0\rangle \otimes(|0,0\rangle+|1,1\rangle)+\frac{1}{\sqrt{2}}(b|1\rangle \otimes(|0,0\rangle+|11\rangle) .\right.
\end{aligned}
$$

$B o b$ at time $t_{0}$

$$
\operatorname{Inf}\left(B o b_{t_{0}}\right)=\left(\left(S_{3}\right)_{t_{0}},\left|\psi^{S_{2}+S_{3}}\right\rangle_{t_{0}}\right)
$$

where

$$
\left|\psi^{S_{2}+S_{3}}\right\rangle_{t_{0}}=\frac{1}{\sqrt{2}}(|0,0\rangle+|1,1\rangle) .
$$

As is well known, the basic goal of quantum teleportation is transmitting a state to a "far" agent by means of a non-local quantum phenomenon. In this particular case, Alice wants to transmit to $B o b$ the state $a|0\rangle+b|1\rangle$ that particle $S_{1}$ has at time $t_{0}$. As expected, the operations performed by Alice in order to obtain this aim will transform her epistemic situation.

\section{Alice at time $t_{1}$}

In the interval $\left[t_{0}, t_{1}\right]$ Alice applies the gate XOR ${ }^{(1,1)}$ to the subsystem $S_{1}+S_{2}$ (accessible to her). As a consequence, we obtain:

$$
\operatorname{Inf}\left(\text { Alice }_{t_{1}}\right)=\left(\left(S_{1}+S_{2}\right)_{t_{1}},\left|\psi^{S}\right\rangle_{t_{1}}\right)
$$

where:

$$
\begin{aligned}
\left|\psi^{S}\right\rangle_{t_{1}} & =\left[\mathrm{XOR}^{(1,1)} \otimes \mathrm{I}^{(1)}\right]\left|\psi^{S}\right\rangle_{t_{0}} \\
& =\frac{1}{\sqrt{2}}\left(a|0\rangle \otimes(|0,0\rangle+|1,1\rangle)+\frac{1}{\sqrt{2}}(b|1\rangle \otimes(|1,0\rangle+|0,1\rangle) .\right.
\end{aligned}
$$

It is worth-while noticing that theoretically Alice is acting on the whole system $S$, while materially she is only acting on the subsystem $S_{1}+S_{2}$ that is accessible to her. 


\section{QUANTUM TELEPORTATION AND QUANTUM EPISTEMIC SEMANTICS}

\section{Alice at time $t_{2}$}

In the interval $\left[t_{1}, t_{2}\right]$ Alice applies the gate Hadamard to the system $S_{1}$ (whose state is to be teleported). Hence, we obtain:

$$
\operatorname{Inf}\left(\text { Alice }_{t_{2}}\right)=\left(\left(S_{1}+S_{2}\right)_{t_{2}},\left|\psi^{S}\right\rangle_{t_{2}}\right)
$$

where:

$$
\begin{aligned}
\left|\psi^{S}\right\rangle_{t_{2}}= & {\left[\sqrt{\mathrm{I}}^{(1)} \otimes \mathrm{I}^{(1)} \otimes \mathrm{I}^{(1)}\right]\left|\psi^{S}\right\rangle_{t_{1}} } \\
= & \frac{1}{\sqrt{2}} \frac{1}{\sqrt{2}}\{[a(|0\rangle+|1\rangle) \otimes(|0,0\rangle+|1,1\rangle)]+[b(|0\rangle-|1\rangle) \otimes(|1,0\rangle+|0,1\rangle)]\} \\
= & \frac{1}{2}[(|0,0\rangle \otimes(a|0\rangle+b|1\rangle))+(|0,1\rangle \otimes(a|1\rangle+b|0\rangle)) \\
& +(|1,0\rangle \otimes(a|0\rangle-b|1\rangle))+(|1,1\rangle \otimes(a|1\rangle-b|0\rangle))] .
\end{aligned}
$$

\section{Alice at time $t_{3}$}

In the interval $\left[t_{2}, t_{3}\right]$ Alice decides to perform a measurement on the subsystem $S_{1}+S_{2}$ (accessible to her). As a consequence (by collapse of the wave function) she will obtain with equal probability $\left(=\frac{1}{4}\right)$ one of the four following quregisters:

1. $\left|\psi_{1}^{S_{t_{3}}}\right\rangle=|0,0\rangle \otimes(a|0\rangle+b|1\rangle)$

2. $\left|\psi_{2}^{S_{t_{3}}}\right\rangle=|0,1\rangle \otimes(a|1\rangle+b|0\rangle)$

3. $\left|\psi_{3}^{S_{t_{3}}}\right\rangle=|1,0\rangle \otimes(a|0\rangle-b|1\rangle)$

4. $\left|\psi_{4}^{S_{t_{3}}}\right\rangle=|1,1\rangle \otimes(a|1\rangle-b|0\rangle)$

We have:

$$
\operatorname{Inf}\left(\text { Alice }_{t_{3}}\right)=\left(\left(S_{1}+S_{2}\right)_{t_{3}},\left|\psi_{i}^{S}\right\rangle_{t_{3}}\right),
$$

where: $\left|\psi_{i}^{S}\right\rangle_{t_{3}}$ is one of the four states considered above.

Notice that after Alice's measurement (at time $t_{3}$ ) the original superposed state $a|0\rangle+b|1\rangle$ of particle $S_{1}$ has disappeared. The state of $S_{1}$ is now:

$$
\left|\psi^{S_{1}}\right\rangle_{t_{3}}=\operatorname{Red}^{1}\left(\left|\psi_{i}^{S}\right\rangle_{t_{3}}\right)
$$

(which is a bit $|x\rangle$ ).

As a consequence of her measurement, Alice also knows the qubit representing the state at time $t_{3}$ of particle $S_{3}$ (accessible to $B o b$ ). In fact, by quantum nonlocality, the state of particle $S_{3}$ has been transformed into one of the four possible qubits:

$$
\left|\varphi_{i}^{S_{3}}\right\rangle_{t_{3}}=\operatorname{Red}^{3}\left(\left|\psi_{i}^{S}\right\rangle_{t_{3}}\right), \quad \text { with } \quad 1 \leq i \leq 4
$$

Apparently, only $\left|\varphi_{1}^{S_{3}}\right\rangle_{t_{3}}$ is $a|0\rangle+b|1\rangle$, the original state of particle $S_{1}$ (accessible to Alice). Anyway, by application of a convenient gate, all states $\left|\varphi_{i}^{S_{3}}\right\rangle_{t_{3}}$ can be transformed into the state $a|0\rangle+b|1\rangle$. We have: 


\section{E. BELTRAMETTI - M. L. DALLA CHIARA - R. GIUNTINI - G. SERGIOLI}

- $\mathrm{I}^{(1)}\left(\left|\varphi_{1}^{S_{3}}\right\rangle_{t_{3}}\right)=a|0\rangle+b|1\rangle$

- $\operatorname{NOT}^{(1)}\left(\left|\varphi_{2}^{S_{3}}\right\rangle_{t_{3}}\right)=a|0\rangle+b|1\rangle$

- $Z^{(1)}\left(\left|\varphi_{3}^{S_{3}}\right\rangle_{t_{3}}\right)=a|0\rangle+b|1\rangle$

- $\operatorname{NOT}^{(1)} Z^{(1)}\left(\left|\varphi_{4}^{S_{3}}\right\rangle_{t_{3}}\right)=a|0\rangle+b|1\rangle$,

where $Z^{(1)}$ is the Pauli matrix

$$
\left(\begin{array}{cc}
1 & 0 \\
0 & -1
\end{array}\right)
$$

In this situation, Alice can give an "order" to Bob, by using a classical communication channel (say, a phone) during the interval $\left[t_{3}, t_{4}\right]$. The order will be:

- "apply I (1)!" (i.e. "don't do anything!"), in the first case.

- "apply NOT ${ }^{(1)}$ !", in the second case.

- "apply $Z^{(1)}$ !", in the third case.

- "apply $\mathrm{NOT}^{(1)} Z^{(1)}$ ! in the fourth case.

Suppose that Bob follows Alice's order in the interval $\left[t_{4}, t_{5}\right]$. His final epistemic situation (at time $t_{5}$ ) will be:

$$
\operatorname{Inf}\left(B o b_{t_{5}}\right)=\left(\left(S_{3}\right)_{t_{5}},\left|\psi^{S_{3}}\right\rangle_{t_{5}}\right),
$$

where $\left|\psi^{S_{3}}\right\rangle_{t_{5}}=a|0\rangle+b|1\rangle$.

Teleportation is now completed. At the end of the process, the original qubit $a|0\rangle+b|1\rangle$ has disappeared for Alice, because at the final time the system $S_{1}$ is storing a classical bit. Bob, instead, has acquired the information $a|0\rangle+b|1\rangle$, which is stored by "his" particle $S_{3}$, whose original state was the mixture:

$$
\operatorname{Red}^{2}\left(\left|\psi^{S_{2}+S_{3}}\right\rangle_{t_{0}}\right)=\operatorname{Red}^{2}\left(\frac{1}{\sqrt{2}}(|0,0\rangle+|1,1\rangle)\right)=\frac{1}{2} P_{0}^{(1)}+\frac{1}{2} P_{1}^{(1)} .
$$

When discussing teleportation, one often stresses that Alice might ignore the qubit $a|0\rangle+b|1\rangle$ that is transmitted to Bob. Such observation (which may appear prima facie somewhat puzzling) can be interpreted as follows.

- At time $t_{0}$ Alice has physical access to particle $S_{1}$;

- Alice knows that the state of $S_{1}$ (at time $t_{0}$ ) is pure: a qubit whose form is $a|0\rangle+b|1\rangle$;

- in spite of this, Alice ignores the actual values of the two amplitudes $a$ and $b$, which are dealt with by her as complex number variables.

One could also say that what Alice knows is not a real qubit, but rather a kind of metaqubit (which ranges over all possible qubits). Such ignorance, however, does not prevent Alice to perform all operations that are needed in 
order to transmit the "real" qubit to Bob. Of course, she can physically act on the subsystem $S_{1}+S_{2}$ (accessible to her) both by applying the convenient material gates and by performing the measurement that determines the final collapse of the wave function. At the same time, she can theoretically calculate the metastates that correspond to the states $\left|\psi^{S}\right\rangle_{t_{1}},\left|\psi^{S}\right\rangle_{t_{2}},\left|\psi^{S}\right\rangle_{t_{3}}$ (where $a$ and $b$ are dealt with as complex number variables).

In this perspective, the epistemic situation of any agent at a given time $t$ should be more adequately dealt with as a triplet:

$$
\operatorname{Inf}\left(\text { Agent }_{t}\right)=(S, \rho, \widetilde{\rho}),
$$

where:

- $S$ is a physical system accessible to Agent $_{t}$;

- $\rho$ is a (pure or mixed) state for a system $S^{\prime}$ such that $S$ is a subsystem of $S^{\prime}$;

- $\tilde{\rho}$ is a metaqumix for $\rho$.

The notion of metaqumix can be precisely defined as follows:

- Let $\rho$ corresponds to the quregister

$$
|\psi\rangle=\sum_{i} a_{i}\left|x_{i_{1}}, \ldots, x_{i_{n}}\right\rangle .
$$

We say that $|\widetilde{\psi}\rangle$ is a metaqumix for $|\psi\rangle$ iff $|\widetilde{\psi}\rangle$ is the result of a uniform substitution of some of the (constant) amplitudes $a_{i}$ with corresponding complex number variables $\widetilde{a_{i}}$.

- Let $\rho$ be the qumix $\sum_{i} w_{i} P_{\left|\psi_{i}\right\rangle}$. We say that $\tilde{\rho}$ is a metaqumix for $\rho$ iff $\widetilde{\rho}$ is the result of a uniform substitution of some of the (constant) weights $w_{i}$ with corresponding real number variables $\widetilde{w_{i}}$.

When $\widetilde{\rho}$ is a metamix for $\rho$, we will also write: $\widetilde{\rho} \bowtie \rho$. Accordingly, in the logical applications we will be entitled to use holistic metamodels $\widetilde{\text { Hol such that }}$ for any sentence $\alpha, \widehat{\operatorname{Hol}}(\alpha)$ is a convenient metaqumix.

\section{A quantum-classical epistemic semantics}

By abstracting from quantum teleportation phenomena we will now propose an epistemic semantics which consists of two components: a classical and a quantum computational one. To this aim, we consider two languages:

1) the quantum computational language $\mathcal{L}^{Q C}$ (introduced in Section 2), whose sentences represent pieces of quantum information, stored by some quantum systems. 


\section{E. BELTRAMETTI - M. L. DALLA CHIARA - R. GIUNTINI - G. SERGIOLI}

2) A classical epistemic language $\mathcal{L}^{E p}$, whose atomic sentences have the following forms:

- $\mathcal{I} \mathbf{a} \alpha$ (the epistemic agent $a$ has a probabilistic information about the sentence $\alpha$ of $\left.\mathcal{L}^{Q C}\right)$;

- $\mathcal{K} \mathbf{a} \alpha$ (the epistemic agent $a$ has a certain knowledge about the sentence $\alpha$ of $\mathcal{L}^{Q C}$ ),

The molecular sentences of $\mathcal{L}^{E p}$ are built up by means of the classical connectives $\left(\neg_{C}, \wedge_{C}\right)$.

We will now introduce the basic notions of our semantics. The central concept is the notion of epistemic model $\mathcal{M}$ of the language $\mathcal{L}^{E p}$.

From an intuitive point of view, $\mathcal{M}$ can be described as a system characterized by the following elements:

1. a time-sequence.

2. A set of epistemic agents evolving in time.

3. Any agent has a truth-perspective that determines his/her idea of truth and probability. We assume that the truth-perspective of each agent is constant in time.

4. Any truth-perspective is associated to a holistic quantum computational model that interprets the sentences of $\mathcal{L}^{Q C}$ as convenient qumixes.

5. At any time any agent has a characteristic epistemic situation, determined by the following elements:

a) a quantum system on which the agent can physically operate. This system can be also regarded as a "material source" for our agent's information.

b) A quantum piece of information $\rho$ that represents the agent's epistemic universe.

c) A metastate $\widetilde{\rho}$ for $\rho$. We can think that $\widetilde{\rho}$ represents a contingent $s u b$ jective ignorance of our agent about the objective piece of information represented by $\rho$.

We will now give the precise definitions of epistemic model and of truth for the sentences of the language $\mathcal{L}^{E p}$.

Definition 4.1 (Epistemic model).

An epistemic model of the language $\mathcal{L}^{E p}$ is a system

where:

$$
\mathcal{M}=(T, \mathrm{Ag}, \text { den, TrPersp, Hol, Inf }),
$$

(1) $T$ is a time-sequence.

(2) $\mathrm{Ag}$ is a set of epistemic agents $\mathfrak{a}$ represented as functions of $t$ in $T$. We will write $\mathfrak{a}_{t}$ instead of $\mathfrak{a}(t)$. 
(3) den is a map that assigns to any name a an agent in Ag. By simplicity, we put: $\operatorname{den}(\mathbf{a})=\mathfrak{a}$.

(4) TrPersp is a map that assigns to any agent $\mathfrak{a}$ a general basis $B_{\mathfrak{a}}$ : the truth-perspective of $\mathfrak{a}$.

(5) Hol is a map that assigns to any $B_{\mathfrak{a}}$ a holistic model of the language $\mathcal{L}^{Q C}$.

(6) Inf is a map that assigns to any $\mathfrak{a}_{t}$ a triplet

$$
\operatorname{Inf}\left(\mathfrak{a}_{t}\right)=\left(S_{\mathfrak{a}_{t}}, \rho_{\mathfrak{a}_{t}}, \widetilde{\rho}_{\mathfrak{a}_{\mathfrak{t}}}\right),
$$

where:

6.1) $S_{\mathfrak{a}_{t}}$ is a physical system, which is supposed to be accessible to $\mathfrak{a}_{t}$.

6.2) $\rho$ represents the state of a physical system $S^{\prime}$ such that $S_{\mathfrak{a}_{t}}$ is a subsystem of $S^{\prime}$. The information $\rho_{\mathfrak{a}_{t}}$ is supposed to be described by a sentence $\gamma_{\mathfrak{a}_{t}}$ such that

$$
\operatorname{Hol}_{B_{\mathfrak{a}}}\left(\gamma_{\mathfrak{a}_{t}}\right)=\rho_{\mathfrak{a}_{t}} .
$$

6.3) $\widetilde{\rho}_{\mathfrak{a}_{t}} \bowtie \rho_{\mathfrak{a}_{t}}$ (i.e. $\widetilde{\rho}_{\mathfrak{a}_{t}}$ is a metaqumix for $\rho_{\mathfrak{a}_{t}}$ ). As a consequence, there is a metamodel $\widetilde{\mathrm{Hol}}_{B_{\mathfrak{a}}}$ such that $\widetilde{\mathrm{Hol}}_{B_{\mathfrak{a}}}\left(\gamma_{\mathfrak{a}_{t}}\right)=\widetilde{\rho}_{\mathfrak{a}_{t}}$.

Definition 4.2 (Truth for atomic sentences).

Let $\mathcal{M}=(T, \mathrm{Ag}$, den, TrPersp, Hol, Inf) be an epistemic model for the language $\mathcal{L}^{E p}$. For any time $t$ of $T$ the truth at time $t$ of an atomic sentence of $\mathcal{L}^{E p}$ is defined as follows:

1) $\models_{t} \mathcal{I} \mathbf{a} \alpha$ iff

- $\alpha$ is a subformula of $\gamma_{\mathfrak{a}_{t}}$.

- $\widetilde{\operatorname{Hol}^{\gamma_{\mathfrak{a}}}} B_{\mathfrak{a}}(\alpha)=\operatorname{Hol}_{B_{\mathfrak{a}}}^{\gamma_{\mathfrak{a}_{t}}}(\alpha)$.

2) $\models_{t} \mathcal{K} \mathbf{a} \alpha$ iff $\models_{t} \mathcal{I} \mathbf{a} \alpha$ and $\mathrm{p}_{B_{\mathfrak{a}}}\left(\operatorname{Hol}_{B_{\mathfrak{a}}}^{\gamma_{\mathfrak{a}}}(\alpha)\right)=1$.

Apparently, the sentence $\mathcal{I} \mathbf{a} \alpha$ is true at time $t$ when the following conditions are satisfied:

a) $\alpha$ is a subformula of the sentence $\gamma$ that describes the epistemic universe of agent $\mathfrak{a}$ at time $t$.

b) Although the subjective epistemic universe of agent $\mathfrak{a}$ at time $t$ may only represent a metainformation, the contextual information of $\alpha$ under $\gamma$ is a genuine information. Hence, one can say that our agent at time $t$ understands the meaning of $\alpha$.

The truth of the sentence $\mathcal{K} \mathbf{a} \alpha$ at time $t$ means that:

a) $\alpha$ is understood by agent $\mathfrak{a}$ at time $t$.

b) Furthermore, $\mathfrak{a}$ assigns probability 1 to the contextual meaning of $\alpha$ un$\operatorname{der} \gamma$. 


\section{E. BELTRAMETTI - M. L. DALLA CHIARA - R. GIUNTINI - G. SERGIOLI}

Definition 4.3 (Truth for molecular sentences).

The definition is given according to the usual rules of classical semantics.

The following lemma illustrates some important properties of our epistemic semantics, which permit us to overcome some crucial shortcomings of most standard approaches to epistemic logics.

\section{LEMMA 4.1.}

1. $\models_{t} \mathcal{K} \mathbf{a} \alpha \curvearrowright \neg C \mathcal{K} \mathbf{a} \neg \alpha$, but not the other way around! Hence, knowledge is consistent at any particular time.

2. $\models_{t} \mathcal{K} \mathbf{a}(\alpha \wedge \beta) \curvearrowright \models_{t} \mathcal{K} \mathbf{a} \alpha$ and $\models_{t} \mathcal{K} \mathbf{a} \beta$, but not the other way around! Hence, knowledge is not generally closed under conjunction.

3. $\models_{t} \mathcal{K} \mathbf{a} \alpha$ and $\alpha \models \mathbf{A b s Q C L} \beta \not \models_{t} \mathcal{K} \mathbf{a} \beta$.

Hence, the unrealistic logical omniscience of epistemic agents is avoided.

Let us now go back to the quantum teleportation phenomena. By simplicity we refer to a situation where Alice knows the amplitudes $a$ and $b$ of the qubit $\left|\psi^{S_{1}}\right\rangle_{t_{0}}$ to be teleported. The teleportation case gives rise to a special example of an epistemic model

$$
\mathcal{M}=(T, \mathrm{Ag}, \text { den, TrPersp, Hol, Inf }),
$$

where:

- $T$ is the time-sequence $t_{0}, \ldots, t_{5}$ (considered in the experiment).

- Ag contains the two individuals Alice (a) and Bob (b), represented as functions of the times $t_{0}, \ldots, t_{5}$.

- The truth-perspective is the canonical general basis $B_{\mathrm{I}}$ both for Alice and Bob.

- All states involved in the teleportation phenomenon are supposed to be described by convenient sentences of the language $\mathcal{L}^{Q C}$. In particular, we assume that $\gamma_{\mathfrak{a}_{t_{0}}}$ has the form

$$
\mathbf{s}_{1}^{t_{0}} \sharp \mathbf{s}_{2}^{t_{0}} \sharp \mathbf{s}_{3}^{t_{0}},
$$

where $\mathbf{s}_{1}^{t_{0}}, \mathbf{s}_{2}^{t_{0}}, \mathbf{s}_{3}^{t_{0}}$ are three atomic sentences that describe the states at time $t_{0}$ of $S_{1}, S_{2}, S_{3}$, respectively. Furthermore:

$$
\operatorname{Hol}_{\mathrm{I}}\left(\gamma_{\mathfrak{a}_{t_{0}}}\right)=\rho_{\mathfrak{a}_{t_{0}}}=(a|0\rangle+b|1\rangle) \otimes \frac{1}{\sqrt{2}}(|0,0\rangle+|1,1\rangle) .
$$

Hence we have:

$$
\begin{aligned}
& \operatorname{Hol}_{I}^{\gamma_{a_{a_{0}}}}\left(\mathbf{s}_{1}^{t_{0}}\right)=a|0\rangle+b|1\rangle . \\
& \operatorname{\gamma ol}_{\mathrm{I} a_{t_{0}}}^{\gamma_{\gamma_{0}}}\left(\mathbf{s}_{2}^{t_{0}}\right)=\frac{1}{2} P_{0}^{(1)}+\frac{1}{2} P_{1}^{(1)} . \\
& \operatorname{Hol}_{\mathrm{I}}^{\gamma_{\mathfrak{a}_{t_{0}}}}\left(\mathbf{s}_{3}^{t_{0}}\right)=\frac{1}{2} P_{0}^{(1)}+\frac{1}{2} P_{1}^{(1)} .
\end{aligned}
$$


As a consequence, we obtain:

$$
\models t_{0} \mathcal{I} \mathbf{a s}_{1}^{t_{0}} ; \quad \not t_{t_{0}} \mathcal{K} \mathbf{a s}_{1}^{t_{0}} .
$$

In other words, at time $t_{0}$ Alice has a probabilistic information and no certain knowledge about the sentence $\mathbf{s}_{1}^{t_{0}}$.

For $B o b$, instead, we have:

$\gamma_{\mathfrak{b}_{t_{0}}}=\mathbf{s}_{2}^{t_{0}} \sharp \mathbf{s}_{3}^{t_{0}}$.

$\mathrm{Hol}_{\mathrm{I}}\left(\gamma_{\mathfrak{b}_{t_{0}}}\right)=\rho_{\mathfrak{b}_{t_{0}}}=\frac{1}{\sqrt{2}}(|0,0\rangle+|1,1\rangle)$.

$\operatorname{Hol}_{\mathrm{I}}^{\gamma_{\mathfrak{b}} t_{0}}\left(\mathbf{s}_{2}^{t_{0}}\right)=\frac{1}{2} P_{0}^{(1)}+\frac{1}{2} P_{1}^{(1)}$.

$\operatorname{Hol}_{\mathrm{I}}^{\gamma_{\mathfrak{b}} t_{0}}\left(\mathbf{s}_{3}^{t_{0}}\right)=\frac{1}{2} P_{0}^{(1)}+\frac{1}{2} P_{1}^{(1)}$.

Hence:

$$
\begin{aligned}
& \models_{t_{0}} \mathcal{I}_{\mathbf{b s}}^{t_{2}} ; \quad \not \nvdash_{t_{0}} \mathcal{K} \mathbf{b} \mathbf{s}_{2}^{t_{0}} . \\
& \models_{t_{0}} \mathcal{I}_{\mathbf{b s}}^{t_{0}} ; \quad \not t_{t_{0}} \mathcal{K} \mathbf{b} \mathbf{s}_{3}^{t_{0}} .
\end{aligned}
$$

This construction can be developed in a similar way for all other times $\left(t_{1}, \ldots, t_{5}\right)$. Supposing that the result of Alice's measurement is the register $|1,1\rangle$, we will finally obtain:

$$
\operatorname{Hol}_{\mathrm{I}}^{\gamma_{\mathfrak{a}_{t_{5}}}}\left(\mathbf{s}_{1}^{t_{5}}\right)=|1\rangle
$$

Hence: $\models_{t_{5}} \mathcal{K} \mathbf{a s}_{1}^{t_{5}}$. At the same time:

$$
\models_{t_{5}} \mathcal{I} \mathbf{b s}_{3}^{t_{5}} ; \quad \not t_{t_{5}} \mathcal{K} \mathbf{b s}_{3}^{t_{5}} .
$$

So far, we have dealt with epistemic operators that are expressed in a classical epistemic language. An interesting question is the following: is it possible to extend the quantum computational language $\mathcal{L}^{Q C}$ to an epistemic language where the epistemic operators are interpreted as special kinds of Hilbert space operations? We discuss this problem in [7.

\section{REFERENCES}

[1] AHARONOV, A.: A simple proof that Toffoli and Hadamard are quantum universal, [arXiv:quant-ph/0301040], 2003.

[2] AHARONOV, A.-KITAEV, A.-NISAN A.: Quantum circuits with mixed states. In: STOC '98: Proceedings of the Thirtieth Annual ACM Symposium on Theory of Computing, ACM Press, 1998, pp. 20-30.

[3] DAlla CHIARA, M. L.-GIUNTINI, R.-FREYTES, H.-LEDDA, A. - SERGIOLI G: The algebraic structure of an approximately universal system of quantum computational gates, Found. Phys. 39 (2009), 559-572.

[4] DALla ChiARA, M. L.-GIUNTINI, R.-FREYTES, H.-LEDDA, A.-LEPORINI, R. -SERGIOLIG: Entanglement as a semantic resource, Found. Phys. 40 (2011), 1494-1518. 


\section{E. BELTRAMETTI - M. L. DALLA CHIARA - R. GIUNTINI - G. SERGIOLI}

[5] DALlA CHIARA, M. L.-GIUNTINI, R.-GREECHIE, R.: Reasoning in Quantum Theory, Kluwer, Dordrecht, 2004.

[6] DALLA CHIARA, M. L.-GIUNTINI, R.-LEPORINI, R.: Logics from quantum computation, Internat. J. Quantum Inform. 3 (2005), 293-337.

[7] BELTRAMETTI, E.-DALLA CHIARA, M. L.-GIUNTINI, R.-LEPORINI, R.SERGIOLI, G.: Epistemic quantum computational structures in a Hilbert-Space Environment, Fund. Inform. 115 (2012), 1-14.

[8] GUDDER, S.: Quantum computational logics, Internat. J. Theoret. Phys. 42 (2003), 39-47.

[9] NIELSEN, M. -CHUANG I.: Quantum Computation and Quantum Information, Cambridge University Press, Cambridge, 2000.

[10] SHI, Y.: Both Toffoli and controlled-Not need little help to do universal quantum computation, [arXiv:quant-ph/0205115], 2002.

Received 27. 6. 2011

Accepted 19. 12. 2011
* Dipartimento di Fisica

Università di Genova

Via Dodecaneso 33

I-16146 Genova

ITALY

E-mail: enrico.beltrametti@ge.infn.it

** Dipartimento di Filosofia

Università di Firenze

Via Bolognese 52

I-50139 Firenze

ITALY

E-mail: dallachiara@unifi.it

*** Dipartimento di Filosofia

e Teoria delle Scienze Umane

Università di Cagliari

Via Is Mirrionis 1

I-09123 Cagliari

ITALY

Corresponding author:

Roberto Giuntini

E-mail: giuntini@unica.it

giuseppe.sergioli@gmail.com 